\title{
Routing and Power Adaptation for Mostly-on Wireless Sensor Networks Applications
}

\author{
Mohamed Adnane Zayane, Riadh Dhaou, André-Luc Beylot \\ University of Toulouse, IRIT-ENSEEIHT \\ 2 rue Charles. Camichel, BP 7122, 31071, Toulouse cedex 7, France \\ \{Mohamed.Zayane, Riadh.Dhaou, Andre-Luc.Beylot\}@enseeiht.fr
}

\begin{abstract}
Wireless sensor networks (WSNs) have received much attention in the last few years especially with regard to routing protocols and transmission power adaptation. The main goal of power adaptation is to improve the performance and the lifetime of WSNs. By selecting the suitable Transmission Power Level (TPL), the battery lifetime of sensor nodes is increased and better overall network performance is achieved. In this paper, we investigate the impact problem of TPLs coupled with static routing in mostly-on many-to-one WSN applications. In such network pattern, all sensor nodes generate and continuously send data to a single sink via single-hop or multi-hop transmissions. The impact of routing coupled to TPLs is evaluated using network metrics such as: Packet Delivery Ratio (PDR), energy consumption, transmission latency.
\end{abstract}

Keywords: WSNs, routing, transmission power adaptation.

\section{INTRODUCTION}

A wireless sensor network (WSN) consists of spatially scattered autonomous sensors to monitor physical or environmental conditions and to cooperatively transmit and route their sensed data through the network to a remote base station (BS) [1]. Different multi-hop routing protocols are designed and implemented for WSNs in order to route sensed data to the BS. Furthermore, transmission power adaptation is aimed at increasing the lifetime and achieving better overall network performance specially in some mostly-on WSN applications. Indeed, for mostly-off WSN it is shown, in [2], that using maximum TPL will increase the lifetime of the WSN. Moreover, an experimental study of the correlation between RSSI/LQI and wireless link quality is presented in [5], where a model to estimate the optimal TPL providing a good PDR was introduced. The authors proposed a feedback based adaptive transmission power control (ATPC) algorithm to dynamically maintain wireless link qualities over time. The authors of [6] proposed an efficient transmission power control algorithm ODTPC that can efficiently reduce the transmission power consumption in WSNs while maintaining good link qualities. The authors of [7] proposed an extension of ODTPC algorithm named MODTPC, which dramatically reduces the energy consumption as compared to ODTPC and ATPC. MODTPC introduces optimization in terms of TPL adjustment for each sensor node of the WSN. In [8], the authors presented an adaptive transmission power control method for WSNs. They propose that the optimization of the transmission power of whole WSN is converted to a shortest path problem based on the so-called "power distance". Each transmitter detects the minimum TPL to reach its neighbor sensor nodes and considers the power levels as "distance" to solve a shortest path problem. Furthermore, Multi-hop static routing protocol has been studied in the context of mostly-off WSNs [2-4]. In the present paper we extend this study to the mostly-on traffic pattern. We investigate the impact of routing coupled to TPLs in WSNs. We have used a mostly-on many-to-one WSN, with sensor nodes deployed in a regular grid topology and generating regular Poisson traffic. The BS of the WSN is located at the left corner of the grid. The goal of the study is to explore the impact of routing policy coupled to TPLs on the performance of such WSNs using three network metrics such as: PDR, Power Consumption and Transmission Latency. The remainder of this paper is as follows. Section 2 describes the multi-hop static routing protocol. Section 3, outlines the simulation setup we used to study the impact of routing and TPLs and presents simulation results. Finally, Section 4 presents conclusions and directions for future work.

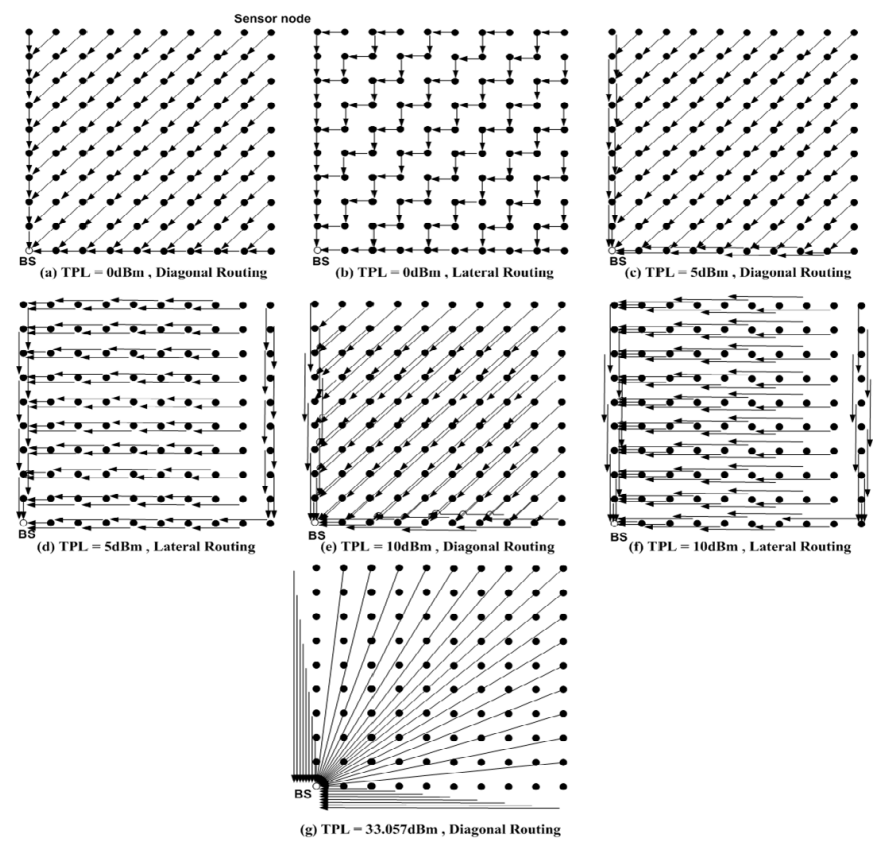

Figure 1. Single-hop and multi-hop static routing patterns

\section{MULTI-HOP STATIC ROUTING PROTOCOL}

Multi-hop Static routing protocol [9] in WSNs is an efficient routing protocol because the routes used by sensor nodes to reach the BS, are pre-calculated in an optimal way (e.g. shortest path), with respect to the radio transmission range of each sensor node. In WSNs using multi-hop static routing policy, each sensor node has a predefined static routing table. This table includes the minimum information of the next reachable hop toward the BS. This means that each sensor node 
must know the predefined route to reach the BS. This routing policy suppresses routing overheads and reduces link losses. Fig. 1 illustrates the single-hop and multi-hop static routing patterns studied in this paper. These routing patterns are based on SPF algorithm and show the shortest paths used to route data. In this study we investigate four TPLs of sensor nodes. For each TPL we used a defined routing pattern stored in each sensor node of the WSN.

\section{PERFORMANCE EVALUATION}

To investigate the impact of routing patterns and TPLs on the network performance of mostly-on WSNs, we performed simulations using the NS-2 tool. We used a mostly-on manyto-one WSN with regular grid topology, well-known tracking static sensor nodes and regular transmitted traffic. The BS is placed at the bottom left corner of the grid. We also used for communication the IEEE 802.15.4 protocol with non beaconenabled mode and enabled Acknowledge. Four TPLs of sensor nodes are simulated and evaluated: $0,5,10$ and $33.057 \mathrm{dBm}$. For each fixed TPL we used different routing patterns: either diagonal routing pattern or lateral routing one. The packets inter-arrival time varies from $0.01 \mathrm{~s}$ to $10 \mathrm{~s}$. We have studied and evaluated three network metrics: the PDR, the power consumption and the transmission latency. The main parameters of the simulation are defined in the Table below:

TABLE I. SimUlation PARAMETERS

\begin{tabular}{|l|l||l|l|}
\hline \multicolumn{1}{|c|}{ Parameters } & \multicolumn{1}{c||}{ Value } & \multicolumn{1}{c|}{ Parameters } & \multicolumn{1}{c|}{ Value } \\
\hline Sensor field & $550 \mathrm{~m} \times 550 \mathrm{~m}$ & MAC layer & IEEE 802.15 .4 \\
\hline Number of sensor nodes & 99 & Buffer size & 50 packets \\
\hline Inter-node distance & $50 \mathrm{~m}$ & Antenna model & OmniAntenna \\
\hline Frequency & $2.4 \mathrm{GHz}$ & Antenna Height & $0.03125 \mathrm{~m}$ \\
\hline Receive power threshold & $1.17086 \mathrm{e}-17 \mathrm{w}$ & Initial energy & $27540 \mathrm{~J}$ \\
\hline Carrier Sensing threshold & $1.17086 \mathrm{e}-17 \mathrm{w}$ & Traffic type & Poisson \\
\hline Capture Packet threshold & $10.0 \mathrm{~dB}$ & Packets size & 62 bytes \\
\hline Radio Propagation & TwoRayGround & Simulation time & $1000 \mathrm{~s}$ \\
\hline
\end{tabular}

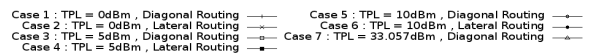

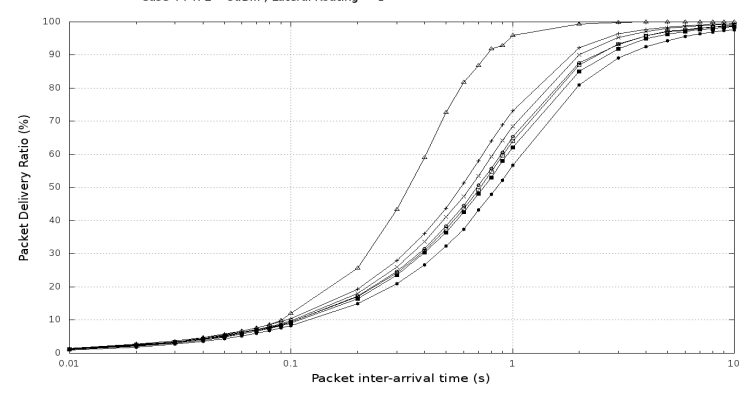

Figure 2. Packet Delivery Ratio

1) Packet Delivery Ratio (PDR). This metric measures the percentage of data packets successfully delivered to the BS compared to packets sent to the network by all sensor nodes. This metric represents the reliability of the routing protocol to deliver the sent data packets. Fig. 2 illustrates the variation of the PDR of the WSN according to packets inter-arrival time for different TPLs and different static routing pattern. For different TPLs and different routing patterns, the PDR of the WSN is improved considerably when the network load decreases because the WSN becomes decreasingly congested. We remark also that, for the TPLs $0 \mathrm{dBm}$ and $5 \mathrm{dBm}$, using multi-hop diagonal routing patterns gives better PDR than using multihop lateral routing patterns. For example, for $1 \mathrm{~s}$ packet interarrival time the PDR varies from $74 \%$ (for diagonal) to $67 \%$ (for lateral). This is due to the increases of the hops number needed to route data packets to the BS. We notice also, that using TPL $0 \mathrm{dBm}$ coupled with multi-hop diagonal routing pattern or multi-hop lateral routing pattern gives better PDR than using TPL $5 \mathrm{dBm}$ coupled with multi-hop diagonal routing pattern or multi-hop lateral routing pattern.

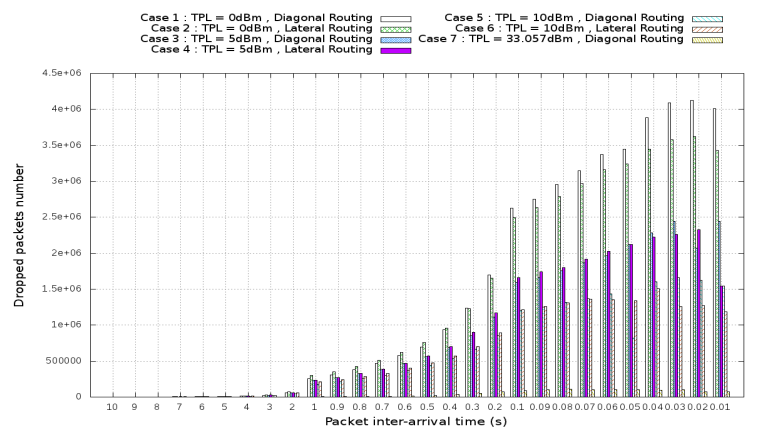

Figure 3. Link Quality Indication drops statistics

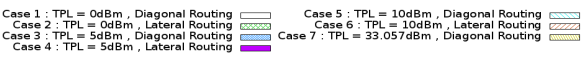

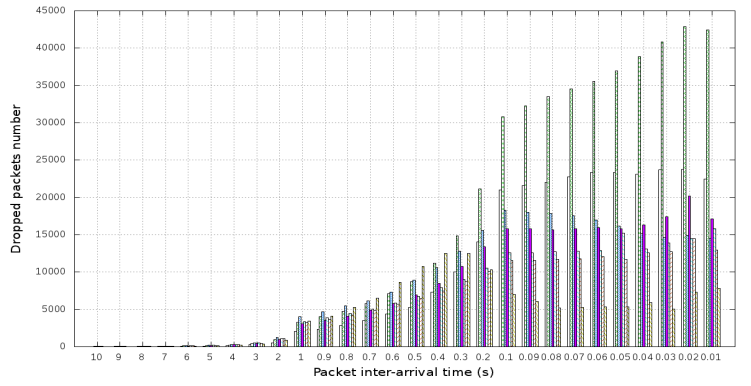

Figure 4. MAC Duplicate packets drops statistics

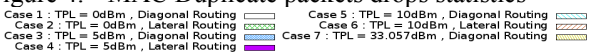

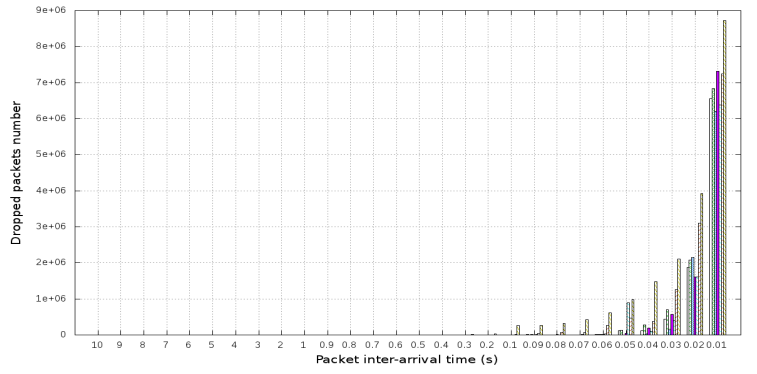

Figure 5. InterFace Queue drops statistics

Packets drop analysis aims at studying the causes of the packets drops and their effect on the network performance of the mostly-on WSN. First of all, we remark that whatever the WSN load, packet drops caused by MAC collision do not occur. The main causes of packets drops are the Link Quality Indicator (LQI), the MAC duplicate packets and the buffer Interface Queue full. Fig. 3, 4 and 5 indicate the drops statistics for different TPLs and different static routing patterns respectively for LQI drops, MAC duplication drops and Interface queue full drops. Indeed, for each data packet received, its LQI is calculated as the signal to noise ratio (SNR) within the channel. If the LQI value is less than the Capture 
packet threshold value which is $10 \mathrm{~dB}$ the packet is dropped otherwise it is accepted. Furthermore, due to non reception of the ACK by the source sensor nodes and to maximum number of frame retries (MaxFrameRetries $=3$ ) of the IEEE 802.15.4 protocol, allowed after a packet transmission failure, duplicate packets will be received by the BS. Duplicate packets are then dropped by the MAC layer of the BS. In addition, when the transmission rate increases, the FIFO queue of the Interface can be full and packets can be dropped. The analysis of fig. 3, 4 and 5, shows that for different TPLs and different routing patterns, respectively LQI-drops, MAC duplication drops and Interface queue drops increase considerably with the increase of network load because the WSN becomes increasingly congested. We also notice, that for low WSN load the main causes of packet drops are LQI-drops and MAC duplication drops, whereas for high WSN load, in addition to the two causes cited above, the saturation of the Interface queue is considerable. We also remark that, the higher the concurrent transmissions rate in the WSN, the lower the opportunity to access the channel for sensor nodes, which increases the probability of queues saturation.

2) Energy Consumption. This metric measures the average amount of energy consumed by each sensor node in the WSN, where the consumed energy of a sensor node is the sum of the energy consumed in transmitting, receiving and idling. Fig. 6 analyzes the average sensor node energy consumption of the mostly-on WSN, versus packets inter-arrival time of sensor nodes. We observe that for different TPLs coupled with different static routing patterns, the average sensor energy consumption decreases with the increase of the packets interarrival time. This is due to the decreasing number of data packets generated by all sensor nodes which should be routed to the BS. We also remark that, for the TPLs $0 \mathrm{dBm}$ and $5 \mathrm{dBm}$ coupled with multi-hop diagonal routing patterns gives better energy consumption than coupled with multi-hop lateral routing patterns. This is due to the increases in the hops number needed to route data packets to the BS. We also notice, that using TPL $0 \mathrm{dBm}$ coupled with multi-hop diagonal or lateral routing patterns gives better energy consumption than using TPL 5dBm.

3) Transmission Latency. This metric measures the average amount of time between the start of disseminating a data packet and its arrival at the BS receiving the data packet. Hence, the transmission latency measures the time performance for the individual message. Fig. 7 illustrates the variation of the average transmission latency according to packets inter-arrival time for different TPLs coupled with different static routing patterns. We note that, for different TPLs and different routing patterns, when the packets inter-arrival time increases the average transmission latency decreases. The greater is the sensor nodes packets inter-arrival time, the less significant is the generated traffic. The WSN will be decreasingly congested. Thus, data packets spend a relatively short delay in the queues. Additionally, retransmissions due to losses are less frequent. We also remark that, using TPL $33.057 \mathrm{dBm}$ coupled with single-hop routing pattern gives better transmission latency than TPLs $0 \mathrm{dBm}, 5 \mathrm{dBm}$ and $10 \mathrm{dBm}$ coupled with multi-hop routing patterns. We also see that, for the TPLs $0 \mathrm{dBm}$ and $5 \mathrm{dBm}$ coupled with multi-hop diagonal routing patterns gives better transmission latency than using multi-hop lateral routing patterns. This is due to the increase in the number of hops needed to route data packets to the BS. We also observe that, increasing the sensor nodes TPL and decreasing the number of hops used by data packets to reach the BS, will decrease the transmission latency.

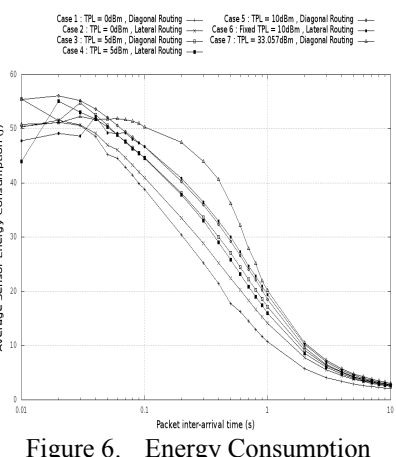

Figure 6. Energy Consumption

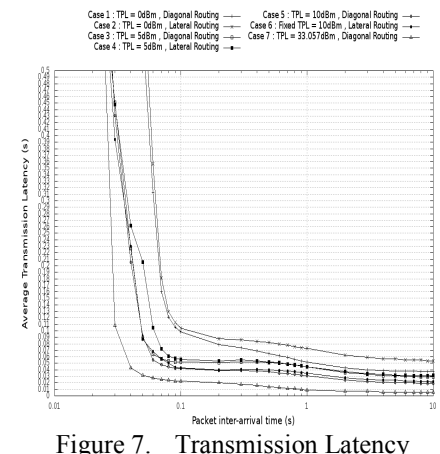

Figure 7. Transmission Latency

\section{CONCLUSION}

In this paper, we have investigated the impact of routing and TPLs on the performance of mostly-on WSNs applications using static routing protocol through several network metrics such as PDR, Energy Consumption and Transmission Latency. Different TPLs of sensor nodes coupled with different routing patterns have been used to perform this study. The simulation results have shown that sensor nodes should adapt their transmission power according to the routing patterns used to route packets to the $\mathrm{BS}$, in order to improve the overall performance of the mostly-on WSN. If sensor nodes can reach the BS by direct transmission, they should use sufficient TPL to transmit packets directly to the BS. Otherwise, they should transmit with a lower TPL that provides the best tradeoff between the length of paths used to route packets to the BS and the collision probability. In future work, we will investigate the impact of load-balancing routing and transmission power adaptation on the performance of mostly-on WSNs applications using static routing protocol.

\section{REFERENCES}

[1] I. F. Akyildiz and al., "Wireless sensor networks: a survey", Computer Networks, Vol. 38, Issue 4, Pages 393-422, 2002.

[2] R. Kacimi, R. Dhaou, A.-L. Beylot, "Energy-balancing Strategies for Lifetime Maximizing in Wireless Sensor Networks", IEEE ICC 2010.

[3] R. Kacimi, R. Dhaou, A.-L. Beylot, "Using energy-efficient wireless sensor network for cold chain monitoring”, IEEE CCNC 2009.

[4] R. Kacimi, R. Dhaou, A.-L. Beylot, "Energy-aware self-organization algorithms for wireless sensor networks", IEEE GLOBECOM 2008.

[5] S. Lin, J. Zhang, L. Gu, T. He, and J. Stankovic, "ATPC: Adaptive Transmission Power Control for WSNs", SenSys 2006.

[6] M. Kim, S. Chang, and Y. Kwon, "ODTPC - On-Demand Transmission Power Control for WSNs”, IEEE ICOIN 2008.

[7] Masood M.M.Y., Ahmed G., Khan N.M. « Modified on demand transmission power control for WSNs », ICICT 2011.

[8] L. Zheng, W. Wang, A. Mathewson, B. o'Flynn, M. Hayes, “An adaptive transmission power control method for WSNs”, ISSC 2010.

[9] K. Akkaya, M. Younis, "A survey on routing protocols for wireless sensor networks", J. of Ad Hoc Networks, Elsevier, p. 325-349, 2005.

[10] L. H. A. Correia and al., "Transmission power control techniques for WSNs", in Computer Networks, Vol. 51, Issue 17, 2007, p 4765-4779. 